\title{
Quantum Fisher information perspective on sensing in anti-PT symmetric systems
}

\author{
J. Wang $\odot,{ }^{1,2}$ D. Mukhopadhyay $\odot,,^{1,2}$ and G. S. Agarwal $\oplus^{1,2,3}$ \\ ${ }^{1}$ Department of Physics and Astronomy, Texas A\&M University, College Station, Texas 77843, USA \\ ${ }^{2}$ Institute for Quantum Science and Engineering and Department of Biological and Agricultural Engineering, \\ Texas A\&M University, College Station, Texas 77843, USA \\ ${ }^{3}$ Department of Biological and Agricultural Engineering, Texas A\&M University, College Station, Texas 77843, USA
}

(Received 12 October 2021; accepted 27 January 2022; published 18 February 2022)

\begin{abstract}
The efficient sensing of weak environmental perturbations via special degeneracies called exceptional points in non-Hermitian systems has attracted enormous attention in the last few decades. However, in contrast to the extensive literature on parity-time (PT) symmetric systems, the exotic hallmarks of anti-PT symmetric systems are only beginning to be realized now. Very recently, a characteristic resonance of vanishing linewidth in anti-PT symmetric systems was shown to exhibit tremendous sensitivity to intrinsic nonlinearities. Given the primacy of sensing in non-Hermitian systems, in general, and the immense topicality of anti-PT symmetry, we investigate the statistical bound to the measurement sensitivity for any arbitrary perturbation in a dissipatively coupled, anti-PT symmetric system. Using the framework of quantum Fisher information and the long-time solution to the full master equation, we analytically compute the Cramér-Rao bound for the system properties such as the detunings and the couplings. As an illustrative example of this formulation, we inspect and reaffirm the role of a long-lived resonance in dissipatively interacting systems for sensing applications.
\end{abstract}

DOI: 10.1103/PhysRevResearch.4.013131

\section{INTRODUCTION}

The Hamiltonian of a physical system characterizes its energy spectrum and time evolution, and is thus of fundamental importance in quantum theory. In nature, all systems are invariably dissipative as they interface with their environment and relax into thermal equilibrium. As a consequence, an open-system formulation in terms of the master equation becomes imperative. Such a description leads to the system dynamics being described in terms of an effective nonHermitian Hamiltonian [1,2]. Over the last few years, certain symmetry properties of these non-Hermitian Hamiltonians have facilitated enhanced sensing of weak perturbations. For instance, the sensing potential of exceptional points (EPs) visà-vis linear perturbations in both parity-time (PT) symmetric and anti-PT symmetric systems have been brought to the fore [3-9]. For example, in a two-mode system, the square-root singularity at an EP engenders a heightened sensitivity in the spectral splitting to perturbative influences. That is to say, the normal mode splitting scales as the square root of the perturbation, which produces a divergent behavior in the firstorder derivative with respect to the perturbation parameter. Recently, in the specific context of anti-PT symmetric systems, a protocol to efficiently detect weak nonlinearities was

Published by the American Physical Society under the terms of the Creative Commons Attribution 4.0 International license. Further distribution of this work must maintain attribution to the author(s) and the published article's title, journal citation, and DOI. also proposed [10]. The scheme exploited a real spectral singularity of the linear response function which strongly inhibits the linewidth of a resonance, thereby drawing out a spectacular response. It was shown that, near the singular point, the nonlinear response $\mathscr{R}$ behaved as $\delta \mathscr{R} / \delta U \propto U^{-5 / 3}$, where $U$ quantifies the strength of Kerr nonlinearity. Unlike PT symmetry, anti-PT symmetry does not require any extrinsic gain, and is predisposed to simple laboratory realization. A wide array of physical systems have been tailored to exhibit anti-PT symmetry in a multitude of settings including, but not limited to, rapidly moving atomic vapor [11], dressed atomic lattices $[12,13]$, driven cold atoms [14,15], laser-cooled atomic ensembles [16], metamaterials [17], nonlinear optical systems [18], waveguides [19,20], electrical $L R C$ resonators [21], diffusive systems [22], coupled cavity-magnon systems [23], and many more, revealing exotic physical phenomena. Recently, Wen et al. [24] demonstrated anti-PT symmetry in a quantum circuit model with three qubits using nuclear spins.

In the context of sensing, it must be acknowledged that the developments heretofore on the sensing potential near special singularities of the system, such as the EP, have been quite remarkable. It is also to be noted that empirical measurements suffer inherently from statistical imprecision, and therefore, uncertainties in the measured response impose fundamental restrictions to the estimation of system parameters. For example, the indelible impact of quantum-limited intensity noise on EP-based sensing protocols was acknowledged in a recent comment by Wiersig [25]. In order to address the inevitability of statistical errors, we bring in a quantum statistical outlook to the problem of sensing, framed and contextualized in anti-PT symmetric configurations. Employing the well-known framework of quantum Fisher information (QFI) [26], we 
formulate the sensitivity measure in terms of the Cramér-Rao bound $[27,28]$, which sets down a theoretical constraint to parameter estimation. The concept of Fisher information was conceived as a tool to quantify the amount of information encoded in an observable quantity about an unknown variable through statistical modeling. With the statistical properties of a quantum system described by its density matrix, the notion was subsequently generalized to quantum formalism [29-38], and has been applied for diverse practical ends, such as for quantum metrology in lossy open systems [39-42]. In this paper, we report the exact precision bound to parameter estimation in anti-PT symmetric systems and thereby provide an overarching statistical framework for the sensing of weak perturbative effects.

The paper is structured as follows. In Sec. II, we briefly overview the basics of anti-PT symmetry from a classical perspective. In Sec. III, we employ the quantum apparatus and show that anti-PT symmetric systems engineered through vacuum-induced dissipative couplings relax into a coherent state in the long-time limit. In Sec. IV, we work out a general formula for the QFI of a coherent state which provides a lower bound to the sensitivity of an arbitrary perturbation variable.

\section{SENSING CAPABILITIES OF CLASSICAL ANTI-PT SYMMETRIC SYSTEMS}

According to the coupled-mode theory in classical optics, the temporal evolution of an open system of two harmonic modes can be described via a $2 \times 2$ non-Hermitian Hamiltonian matrix $\mathscr{H}$. In response to any electromagnetic excitation, the dynamics of the system, in the rotating frame of the drive of frequency $\omega_{d}$, can be encoded as

$$
\left(\begin{array}{c}
\dot{\alpha_{0}} \\
\dot{\beta_{0}}
\end{array}\right)=-i \mathscr{H}\left(\begin{array}{l}
\alpha_{0} \\
\beta_{0}
\end{array}\right)+\mathcal{E}\left(\begin{array}{l}
1 \\
0
\end{array}\right),
$$

where $\left(\alpha_{0}, \beta_{0}\right)$ signifies the classical amplitudes of the two modes, and $\mathcal{E}$ is some generalized Rabi frequency. The generic $2 \times 2$ matrix $\mathscr{H}$ lends itself to two very interesting symmetries, namely PT symmetry, which is defined by the condition $[P T, H]=0$, and anti-PT symmetry, which subscribes to $\{P T, H\}=0$. Throughout this paper, we tailor our analysis around the constraint of anti-PT symmetry as we set forth some information-theoretic restrictions to sensing in systems exhibiting anti-PT symmetry. Such symmetry can be reconciled with the parameter description $\mathscr{H}_{11}=\Delta-i \gamma, \mathscr{H}_{22}=$ $-\Delta-i \gamma, \mathscr{H}_{12}=\mathscr{H}_{21}=-i \Gamma$, where $(\Delta, \gamma, \Gamma)$ are all real, and $\Delta$ is the frequency of the mode $\alpha-\omega_{d}$. The coherenceinducing off-diagonal elements of $\mathscr{H}$ characterize a purely dissipative form of interaction between the two modes. The diagonal terms indicate equal damping rates but opposite detunings in the two modes. The eigenvalues for this Hamiltonian are obtained to be $-i \gamma \pm \sqrt{\Delta^{2}-\Gamma^{2}}$ for $|\Delta|>\Gamma$ and $-i \gamma \pm i \sqrt{\Gamma^{2}-\Delta^{2}}$ (broken anti-PT) for $|\Delta|<\Gamma$. The points of transition $\Delta= \pm \Gamma$, where the eigenvalues coalesce, are known as exceptional points, which have been found useful in the context of sensing. In addition to that, such systems possess a real singularity at $\Delta \rightarrow 0, \gamma \rightarrow \Gamma$, which has immense sensing potential, allowing efficient detection of both linear and nonlinear perturbations in the system. The key measurement parameter, in this case, is the residual response of the system characterized by the amplitudes $\alpha_{0}(\varepsilon)$ and $\beta_{0}(\varepsilon)$, assumed to be functions of a perturbation $\varepsilon$. Subject to the fulfillment of the stability criterion, viz., $\gamma>\Gamma$, the system reduces to a steady state in the long-time limit, yielding the amplitudes

$$
\begin{aligned}
& \alpha_{0}=-i \frac{\mathscr{H}_{22}}{\operatorname{det} \mathscr{H}} \mathcal{E}=-\frac{\gamma-i \Delta}{\Gamma^{2}-\Delta^{2}-\gamma^{2}} \mathcal{E}, \\
& \beta_{0}=i \frac{\mathscr{H}_{21}}{\operatorname{det} \mathscr{H}} \mathcal{E}=\frac{\Gamma}{\Gamma^{2}-\Delta^{2}-\gamma^{2}} \mathcal{E} .
\end{aligned}
$$

In realistic scenarios, however, there would be some discord with the watertight conditions of anti-PT symmetry, no matter how small the error can be made. Since the ensuing mode amplitudes are proportional to $\mathcal{E}$ and sensitive to the system parameters $(\Delta, \gamma, \Gamma)$, the application of a probe field yields nonzero values of the derivatives $\frac{\partial\left(\alpha_{0}, \beta_{0}\right)}{\partial(\Delta, \gamma, \Gamma)}$. This gives us a way of sensing any small perturbations to these parameters. Let us consider, for instance, the particular case when one of the frequency detunings is zero but there is only a small mismatch in the magnitudes of the two detunings, i.e., $\mathscr{H}_{11}=-i \gamma$ and $\mathscr{H}_{22}=-s-i \gamma$, where $s$ is a small parameter. One could get an estimate of this mismatch by measuring the complex amplitudes

$$
\begin{aligned}
& \alpha_{0}(s)=-\frac{\gamma-i s}{\Gamma^{2}+i s \gamma-\gamma^{2}} \mathcal{E}, \\
& \beta_{0}(s)=\frac{\Gamma}{\Gamma^{2}+i s \gamma-\gamma^{2}} \mathcal{E} .
\end{aligned}
$$

The pertinent sensitivities could then be obtained in terms of

$$
\begin{aligned}
\frac{\partial \alpha_{0}(s)}{\partial s} & =\frac{i \Gamma^{2}}{\left(\Gamma^{2}+i s \gamma-\gamma^{2}\right)^{2}} \mathcal{E}, \\
\frac{\partial \beta_{0}(s)}{\partial s} & =\frac{-i \Gamma \gamma}{\left(\Gamma^{2}+i s \gamma-\gamma^{2}\right)^{2}} \mathcal{E} .
\end{aligned}
$$

Clearly, the coherence-induced singularity in the limit $\gamma \rightarrow \Gamma$ gets translated into an infinite theoretical potential for sensing any arbitrary perturbation around this singularity. Supposing that $\gamma=(1+\xi) \Gamma$ for $\xi \ll 1$, both the derivatives would scale as $1 / s^{2}$, if $\xi \ll s / \Gamma$. This would imply an augmented sensing capability around this point. Alternatively, there could be some weak dispersive coupling between $a$ and $b$, which modifies the off-diagonal terms in $\mathscr{H}$ into $\mathscr{H}_{12}=\mathscr{H}_{21}=$ $g-i \Gamma$, where $g \ll \Gamma$. An example of this case from integrated photonics would be a string of two dipolar emitters embedded onto a waveguide and separated by a distance that is scalewise similar to the resonant wavelength. Following the same approach as above, we can compute the corresponding sensitivities as

$$
\begin{aligned}
& \frac{\partial \alpha_{0}(g)}{\partial g}=2 i \frac{(\Gamma+i g)(\gamma-i \Delta)}{\left[(\Gamma+i g)^{2}-\Delta^{2}-\gamma^{2}\right]^{2}} \mathcal{E}, \\
& \frac{\partial \beta_{0}(g)}{\partial g}=-i \frac{(\Gamma+i g)^{2}+\Delta^{2}+\gamma^{2}}{\left[(\Gamma+i g)^{2}-\Delta^{2}-\gamma^{2}\right]^{2}} \mathcal{E} .
\end{aligned}
$$

Once again, for $\xi \ll g / \Gamma$, the derivatives scale as $1 / g^{2}$ in the limit $\Delta \rightarrow 0$. Both these observations are, however, not only classical but also free from the nuance of statistical considerations. Note further that typically in experiments one would 
measure $\frac{\partial\left|\alpha_{0}\right|^{2}}{\partial g}$, etc., i.e., phase information is not measured. In the subsequent sections, we delve into the quantum statistical framework for treating anti-PT symmetric systems and examine the sensing scheme from the perspective of the QFI.

\section{THE QUANTUM MODEL: STEADY-STATE RESPONSE}

The sensitivity bounds, termed as the Cramér-Rao bounds, pertaining to precision measurements are furnished in terms of the statistical probability distributions and the Fisher information. For quantum systems, we need to introduce the QFI and thus we need a quantum description of the anti-PT symmetric system. In the quantum mechanical picture, the dynamics of a noninteracting two-mode system is underpinned by the Hamiltonian operator

$$
\mathcal{H} / \hbar=\omega_{a} a^{\dagger} a+\omega_{b} b^{\dagger} b
$$

where $\omega_{a}$ and $\omega_{b}$ denote the bare resonances of the individual modes $a$ and $b$. If mode $a$ is now irradiated by a laser drive of Rabi frequency $\mathcal{E}$, the interaction Hamiltonian can be reduced to the form

$$
\mathcal{H}_{\text {int }} / \hbar=i\left(\mathcal{E} e^{-i \omega_{d} t} a^{\dagger}-\mathcal{E}^{*} e^{i \omega_{d} t} a\right) .
$$

To stamp out the harmonic time variation in the interaction, it is convenient to rotate variables as $(a, b) \rightarrow(a, b) e^{-i \omega_{d} t}$, leading to a new Hamiltonian

$$
\mathcal{H}_{\mathrm{eff}} / \hbar=\Delta_{a} a^{\dagger} a+\Delta_{b} b^{\dagger} b+i\left(\mathcal{E} a^{\dagger}-\mathcal{E}^{*} a\right) .
$$

Now, the choice $\Delta_{a}=-\Delta_{b}=\Delta$ would entail a partial fulfillment of the criteria for anti-PT symmetry. In addition to this, both the modes could be made to interface with dissipative reservoirs, which can be appropriately addressed in the master equation formalism. If both the modes decay at identical rates, anti-PT symmetry would be consummated. We assume, for simplicity, thermalized reservoirs at zero temperature instill vacuum noise into the hybrid system. While the noise terms average out to zero, an effective dissipative coupling is introduced between $a$ and $b$. For a system with purely dissipative coupling, the master equation can be expressed as

$$
\frac{d \rho}{d t}=-\frac{i}{\hbar}\left[\mathcal{H}_{\mathrm{eff}}, \rho\right]+\kappa \mathcal{L}(a) \rho+\kappa \mathcal{L}(b) \rho+2 \Gamma \mathcal{L}(c) \rho,
$$

where $\kappa$ stands for the intrinsic damping rate of either of the modes into its local heat bath, $\mathcal{L}$ is the Liouvillian operator defined by $\mathcal{L}(\alpha) \rho=2 \alpha \rho \alpha^{\dagger}-\alpha^{\dagger} \alpha \rho-\rho \alpha^{\dagger} \alpha$ for any annihilation operator $\alpha$, and $c=(1 / \sqrt{2})(a+b)$ is the jump operator corresponding to symmetrical couplings of the modes to a shared reservoir. This equation holds under the approximation that the phase delay due to light propagation from one mode to another is an integral multiple of $2 \pi$. We first bring out the correspondence between the quantum mechanical mean values and the classical amplitudes in the steady state. Since $\langle\dot{a}\rangle=\operatorname{Tr}(\dot{\rho} a)$, the preceding equation naturally dictates the dynamics of the mean values, which, upon simplification, reduces to a form commensurate with Eq. (1),

$$
\left(\begin{array}{l}
\langle\dot{a}\rangle \\
\langle\dot{b}\rangle
\end{array}\right)=-i \mathscr{H}\left(\begin{array}{l}
\langle a\rangle \\
\langle b\rangle
\end{array}\right)+\mathcal{E}\left(\begin{array}{l}
1 \\
0
\end{array}\right),
$$

where $\mathscr{H}=\left(\begin{array}{cc}\Delta-i(\kappa+\Gamma) & -i \Gamma \\ -i \Gamma & -\Delta-i(\kappa+\Gamma)\end{array}\right)$. The identification $\gamma=\kappa+\Gamma$ establishes perfect parity with the classical result.

Next, we derive the steady state which would accord not only the solutions to the mean values but also the relevant quantum fluctuations. This would be achieved by solving for the full density matrix of the system. Assuming that a steady state exists, this can be worked out by letting $\dot{\rho}=0$. To that end, we first argue that the excitation term $\mathcal{H}_{\text {int }}$ gets reflected as a two-mode displacement operation [43], underscored by the transformation $(a, b) \rightarrow D\left(\alpha_{0}, \beta_{0}\right)(a, b) D^{\dagger}\left(\alpha_{0}, \beta_{0}\right)=$ $(\mathcal{A}, \mathcal{B})=\left(a-\alpha_{0}, b-\beta_{0}\right)$, where $\left(\alpha_{0}, \beta_{0}\right)=\left(\langle a\rangle_{\mathrm{ss}},\langle b\rangle_{\mathrm{ss}}\right)$ and $D(\alpha, \beta)=\exp \left(\alpha_{0} a^{\dagger}-\alpha_{0}^{*} a\right) \exp \left(\beta_{0} b^{\dagger}-\beta_{0}^{*} b\right)$, and $\left(\alpha_{0}, \beta_{0}\right)$ are given by

$$
\left(\begin{array}{c}
\alpha_{0} \\
\beta_{0}
\end{array}\right)=\left(\begin{array}{c}
\langle a\rangle_{\mathrm{ss}} \\
\langle b\rangle_{\mathrm{ss}}
\end{array}\right)=-i \mathscr{H}^{-1}\left(\begin{array}{c}
\mathcal{E} \\
0
\end{array}\right) .
$$

This is because such a transformation dispels the driving term in the master equation, and casts Eq. (9) into the form

$$
\begin{aligned}
\frac{d \rho}{d t}= & -\frac{i \Delta}{\hbar}\left[a^{\dagger} a-b^{\dagger} b, \rho\right]+\kappa \mathcal{L}(\mathcal{A}) \rho \\
& +\kappa \mathcal{L}(\mathcal{B}) \rho+2 \Gamma \mathcal{L}(C) \rho \\
= & -(\gamma+i \Delta)\left[\mathcal{A}^{\dagger}, \mathcal{A} \rho\right]-(\gamma-i \Delta)\left[\mathcal{B}^{\dagger}, \mathcal{B} \rho\right] \\
& -\Gamma\left[\mathcal{A}^{\dagger}, \mathcal{B} \rho\right]-\Gamma\left[\mathcal{B}^{\dagger}, \mathcal{A} \rho\right]+\text { H.c. },
\end{aligned}
$$

with H.c. denoting the Hermitian conjugate, and $C=$ $(1 / \sqrt{2})(\mathcal{A}+\mathcal{B})$. Now, given the uniqueness of the steady state, we obtain a solution embodied by the conditions

$$
\begin{gathered}
\mathcal{A} \rho=\rho \mathcal{A}^{\dagger}=0, \\
\mathcal{B} \rho=\rho \mathcal{B}^{\dagger}=0,
\end{gathered}
$$

which are conformable only with the bimodal vacuum $\rho=$ $\left|0_{\mathcal{A}}, 0_{\mathcal{B}}\right\rangle\left\langle 0_{\mathcal{A}}, 0_{\mathcal{B}}\right|$ in the displaced representation. Reverting to the original representation, we obtain a coherent state

$$
\rho=\left|\alpha_{0}, \beta_{0}\right\rangle\left\langle\alpha_{0}, \beta_{0}\right|
$$

as our solution to the anti-PT symmetric master equation. It is to be borne in mind that coherent states belong to the family of Gaussian states and contain all their statistical fluctuations of the system, for example, the excitations of the mode $a$ will have a Poisson distribution. In view of Eq. (13), it follows that a calculation of the mean values $\langle a\rangle$ and $\langle b\rangle$ with respect to this coherent state coincides with the classical result laid out in Eq. (2).

The above state can also be inferred from the reformulated master equation expressed in terms of the corresponding phase-space distribution $P(\alpha, \beta)$ for a two-mode system. Following the procedure spelled out in Chap. 10.2 of Ref. [43], we deduce an equivalent master equation in the $P$ representation as

$$
\frac{\partial P}{\partial t}=\frac{\partial}{\partial \alpha}[u(\alpha, \beta) P]+\frac{\partial}{\partial \beta}[v(\alpha, \beta) P]+\text { c.c. },
$$


where $u(\alpha, \beta)=(\gamma+i \Delta) \alpha+\Gamma \beta-\mathcal{E}, \quad v(\alpha, \beta)=\Gamma \alpha+$ $(\gamma-i \Delta) \beta$, and $P(\alpha, \beta)$ is defined as

$$
\rho=\iint P(\alpha, \beta)|\alpha, \beta\rangle\langle\alpha, \beta| d^{2} \alpha d^{2} \beta,
$$

and c.c. stands for the complex conjugate. In the steady state, as $t \rightarrow \infty$, we require $\frac{\partial P}{\partial t}=0$. This provides the quantum steady-state solution for the $P$ function to be

$$
P(\alpha, \beta)=\mathscr{N} \delta^{(2)}(u(\alpha, \beta)) \delta^{(2)}(v(\alpha, \beta)),
$$

where $\delta(\cdot)$ represents the Dirac delta function and $\mathscr{N}$ is some normalization constant, since $P$ must satisfy $\iint P(\alpha, \beta) d^{2} \alpha d^{2} \beta=1$. Considering the linearity of the functions $u$ and $v$, this is equivalent to

$$
P(\alpha, \beta)=\delta^{(2)}\left(\alpha-\alpha_{0}\right) \delta^{(2)}\left(\beta-\beta_{0}\right),
$$

where $\alpha_{0}=-\frac{(\gamma-i \Delta)}{\Gamma^{2}-\Delta^{2}-\gamma^{2}} \mathcal{E}$ and $\beta_{0}=\frac{\Gamma}{\Gamma^{2}-\Delta^{2}-\gamma^{2}} \mathcal{E}$ is the solution to $u\left(\alpha_{0}, \beta_{0}\right)=v\left(\alpha_{0}, \beta_{0}\right)=0$. Substituting this into Eq. (15), we find that the quantum steady state of this driven anti-PT symmetric system must be a coherent state $\left|\alpha_{0}, \beta_{0}\right\rangle\left\langle\alpha_{0}, \beta_{0}\right|$. The coherent nature of this state would remain valid even for small perturbations to any of the system parameters, as long as the system remains purely dissipative. However, the amplitudes $\left(\alpha_{0}, \beta_{0}\right)$ would get modified as per the constraint of Eq. (12). In particular, if the two modes address each other through a weak dispersive interaction $\hbar g\left(a^{\dagger} b+a b^{\dagger}\right)$, the solutions to the response can be found by assigning an imaginary offset to $\Gamma$, i.e., by letting $\Gamma \rightarrow \Gamma+i g$.

\section{THE QUANTUM CRAMÉR-RAO BOUND FOR SENSITIVITY}

As proved in Sec. III, the steady state for an anti-PT symmetric quantum system is a two-mode coherent state that can be written as $\left|\alpha_{0}, \beta_{0}\right\rangle\left\langle\alpha_{0}, \beta_{0}\right|$, where $\alpha_{0}=-\frac{(\gamma-i \Delta)}{\Gamma^{2}-\Delta^{2}-\gamma^{2}} \mathcal{E}$ and $\beta_{0}=\frac{\Gamma}{\Gamma^{2}-\Delta^{2}-\gamma^{2}} \mathcal{E}$ are the long-time responses in the two modes induced by the external field. Thus, information about any arbitrary perturbations to the system parameters would be encoded in the complex amplitudes $\alpha$ and $\beta$, and as such, perturbative effects can be sensed via measurements of the response functions. To put this into an informationtheoretic perspective, we inspect the Cramér-Rao bound for the measurement uncertainty in the estimation of a perturbation parameter. The problem can, in fact, be solved in a general way, and applied to any unknown parameter $\varepsilon$, as long as the response functions $\alpha_{0}(\varepsilon)$ and $\beta_{0}(\varepsilon)$ are exactly known.

To start off, we briefly recapitulate the basic concepts of QFI. In the estimation of an unknown parameter $\varepsilon$, the Cramér-Rao bound reads $\delta \varepsilon \geqslant \sqrt{F_{Q}^{-1}(\varepsilon)}$, where $\delta \varepsilon$ symbolizes the measurement uncertainty or sensitivity of $\varepsilon$, and $F_{Q}(\varepsilon)=\operatorname{Tr}\left[\rho L^{2}\right]$ is the QFI for $\varepsilon$, pertaining to a system with density matrix $\rho$. The symmetric logarithmic derivative (SLD) matrix $L$ is calculated from the equation $\frac{\partial \rho}{\partial \varepsilon}=\frac{1}{2}(L \rho+\rho L)$. Since we have proved that the anti-PT symmetric system goes over into a coherent state in the long-time limit, we work out the algebra for the appropriate steady state. The corresponding density matrix can be expanded out on the basis of Fock states as

$$
\rho=e^{-\left|\alpha_{0}\right|^{2}} e^{-\left|\beta_{0}\right|^{2}} \sum_{m n p q} \frac{\alpha_{0}^{m} \beta_{0}^{n} \alpha_{0}^{* p} \beta_{0}^{* q}}{\sqrt{m ! n ! p ! q !}}|m, n\rangle\langle p, q| .
$$

Since each of the parameters $\left(\alpha_{0}, \beta_{0}\right)$ is a function of $\varepsilon$, we can solve directly for the derivative $\frac{\partial \rho_{m n, p q}}{\partial \varepsilon}$ in terms of the $\frac{\partial \alpha_{0}}{\partial \varepsilon}$ and $\frac{\partial \beta_{0}}{\partial \varepsilon}$. Note that $\alpha_{0}, \beta_{0}$ are complex numbers. Therefore, expressing them as $\alpha_{0}=\left|\alpha_{0}\right| e^{i \theta_{1}}, \beta_{0}=\left|\beta_{0}\right| e^{i \theta_{2}}$, we find the relation

$$
\begin{aligned}
\frac{\partial \rho_{m n, p q}}{\partial \varepsilon}= & {\left[m\left(\frac{\partial \ln \left|\alpha_{0}\right|}{\partial \varepsilon}+i \frac{\partial \theta_{1}}{\partial \varepsilon}\right)+n\left(\frac{\partial \ln \left|\beta_{0}\right|}{\partial \varepsilon}+i \frac{\partial \theta_{2}}{\partial \varepsilon}\right)\right.} \\
& +p\left(\frac{\partial \ln \left|\alpha_{0}\right|}{\partial \varepsilon}-i \frac{\partial \theta_{1}}{\partial \varepsilon}\right)+q\left(\frac{\partial \ln \left|\beta_{0}\right|}{\partial \varepsilon}-i \frac{\partial \theta_{2}}{\partial \varepsilon}\right) \\
& \left.-2\left|\alpha_{0}\right| \frac{\partial\left|\alpha_{0}\right|}{\partial \varepsilon}-2\left|\beta_{0}\right| \frac{\partial\left|\beta_{0}\right|}{\partial \varepsilon}\right] \rho_{m n, p q},
\end{aligned}
$$

which can be analyzed by resolving them into three parts, defined below:

$$
\begin{aligned}
& -2\left(\left|\alpha_{0}\right| \frac{\partial\left|\alpha_{0}\right|}{\partial \varepsilon}+\left|\beta_{0}\right| \frac{\partial\left|\beta_{0}\right|}{\partial \varepsilon}\right) \rho_{m n, p q} \\
& =\frac{1}{2}\left(L_{1} \rho+\rho L_{1}\right)_{m n, p q}, \\
& {\left[(m+p) \frac{\partial \ln \left|\alpha_{0}\right|}{\partial \varepsilon}+(n+q) \frac{\partial \ln \left|\beta_{0}\right|}{\partial \varepsilon}\right] \rho_{m n, p q}} \\
& \quad=\frac{1}{2}\left(L_{2} \rho+\rho L_{2}\right)_{m n, p q}, \\
& i\left[(m-p) \frac{\partial \theta_{1}}{\partial \varepsilon}+(n-q) \frac{\partial \theta_{2}}{\partial \varepsilon}\right] \rho_{m n, p q} \\
& \quad=\frac{1}{2}\left(L_{3} \rho+\rho L_{3}\right)_{m n, p q} .
\end{aligned}
$$

Following the method discussed in Ref. [44], we solve the equations for the individual components separately. In other words, the corresponding SLD gets split as

$$
L=L_{1}+L_{2}+L_{3},
$$

where

$$
\begin{aligned}
& L_{1}=-2\left|\alpha_{0}\right| \frac{\partial\left|\alpha_{0}\right|}{\partial \varepsilon}-2\left|\beta_{0}\right| \frac{\partial\left|\beta_{0}\right|}{\partial \varepsilon}, \\
& L_{2}=2 \frac{\partial \ln \left|\alpha_{0}\right|}{\partial \varepsilon}\left(a^{+} a\right)+2 \frac{\partial \ln \left|\beta_{0}\right|}{\partial \varepsilon}\left(b^{+} b\right), \\
& L_{3}=2 i \frac{\partial \theta_{1}}{\partial \varepsilon}\left[a^{+} a, \rho\right]+2 i \frac{\partial \theta_{2}}{\partial \varepsilon}\left[b^{+} b, \rho\right] .
\end{aligned}
$$

This precise form of $L$ pins down the QFI and the corresponding sensitivity limit. Invoking the definition $F_{Q}(\varepsilon)=\operatorname{Tr}\left[\rho L^{2}\right]$ we conclude that

$$
F_{Q}(\varepsilon)=4\left|\frac{\partial \alpha_{0}}{\partial \varepsilon}\right|^{2}+4\left|\frac{\partial \beta_{0}}{\partial \varepsilon}\right|^{2}
$$

The same result can also be obtained using the pure state QFI method as shown in the Appendix. It may be noted that the pure state result of Ref. [45] itself makes use of the 
(a)

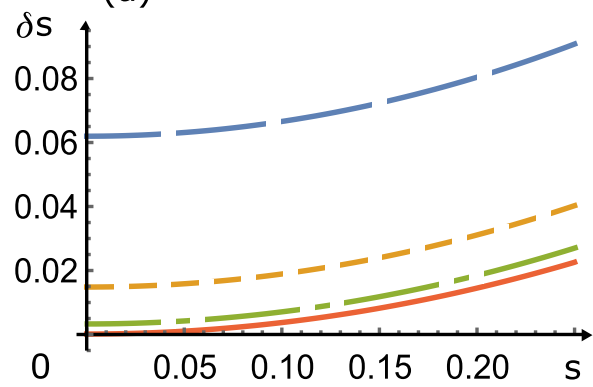

(b)

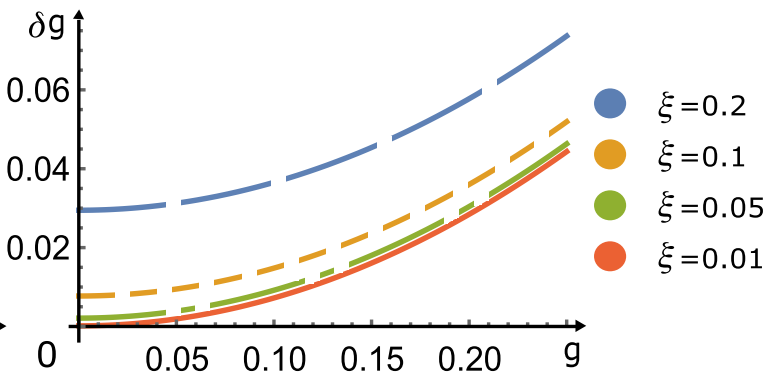

FIG. 1. The Cramér-Rao bounds for measurement uncertainties as $\gamma \rightarrow \Gamma$, with $\mathcal{E} / \Gamma=1$, scaled in units of $\Gamma$ : (a) $\delta s$ for the sensing of detuning mismatch $s$ for different values of $\xi$; (b) $\delta g$ for the sensing of the dispersive coupling strength $g$, at $\Delta=0.1 \Gamma$. The quantity $\xi$, defined as $\xi=(\gamma-\Gamma) / \Gamma$, is a dimensionless measure of the rate of extraneous damping.

logarithmic derivatives. The above expression is absolutely general, regardless of the specifics of the response function $\left(\alpha_{0}(\varepsilon), \beta_{0}(\varepsilon)\right)$. Thus, the QFI, as well as the sensitivity for the system parameters, both depend on the derivatives $\left|\frac{\partial \alpha_{0}}{\partial \varepsilon}\right|$ and $\left|\frac{\partial \beta_{0}}{\partial \varepsilon}\right|$. This result is specific to an anti-PT symmetric mode hybridization, when the interposing reservoir has relaxed into the vacuum state at zero temperature. Apropos of our problem, where the coherent state $\left|\alpha_{0}, \beta_{0}\right\rangle\left\langle\alpha_{0}, \beta_{0}\right|$ encapsulates the statistical properties of the measured quantities, we can model the detuning mismatch $s$ or the weak coupling $g$ as a perturbation. Figures 1(a) and 1(b) depict the sensitivity bounds for these parameters for a set of varying decay rates. For progressively smaller values of the intrinsic damping rate, the perturbations can be sensed with incrementally larger efficacy near the singular point where both the detunings approach zero.

We now discuss our key result Eq. (24) on the QFI and what the experiments typically measure [3-9]. We first note that so far QFI-related concepts have not been used in the context of the anti-PT symmetric systems since the experiments have measured quantities such as $\left|\alpha_{0}\right|^{2}$ to determine the sensitivity to small perturbations. These measurements do not use any statistical measures. Thus typical measurements would use $\frac{\partial\left|\alpha_{0}\right|^{2}}{\partial \varepsilon}$, etc. The whole point about the use of the QFI is to find what is the best estimate of the parameter. For best estimates, quantum fluctuations cannot be avoided. Thus our result (24) for the best possible estimate has contributions from both amplitude and phase, i.e.,

$$
F_{Q}(\varepsilon)=4\left(\frac{\partial|\alpha|}{\partial \epsilon}\right)^{2}+4\left(\frac{\partial|\beta|}{\partial \epsilon}\right)^{2}+4\left(\frac{\partial \theta_{1}}{\partial \epsilon}\right)^{2}|\alpha|^{2}+4\left(\frac{\partial \theta_{2}}{\partial \epsilon}\right)^{2}|\beta|^{2},
$$

where $\theta_{1}\left(\theta_{2}\right)$ is the phase of $\alpha_{0}\left(\beta_{0}\right)$. Clearly, for best measurement, we need to measure both amplitude and phase. In quantum metrology it is well known that when one has to find an optimum measurement to saturate the Cramér-Rao bound based on QFI, one would need to make phase measurements as well, which generally are more involved. Another possibility is to do homodyne measurements to obtain the two quadratures $\operatorname{Re}\left[\alpha_{0}\right]$ and $\operatorname{Im}\left[\alpha_{0}\right]$ which are fairly common in the quantum optics community.

\section{CONCLUSIONS}

In summary, we have offered an information-theoretic insight into the subject of sensing in anti-PT symmetric systems where the coupling between the participating modes is produced by a common vacuum. We invoked the Fisher information theory as a statistical tool to circumscribe the theoretical precision of a single-parameter measurement in terms of the Cramér-Rao bound, which serves as a well-rounded metric for the sensitivity. We argued that anti-PT symmetric systems decay into a coherent state in the long-time limit, which renders the QFI calculation tractable. Then, by treating the steady-state response functions as sensing signals, we derived an exact statistical expression for the sensitivity of the perturbation variable. To exemplify the role of the QFI, we applied this approach to the sensing of a detuning mismatch as well as that of weak coherent coupling between the two modes around a real singularity of the hybrid system. The vanishingly small sensitivity bounds enabled by the emergence of a long-lived resonance reinforced the impact of a strong vacuum-induced coherence on precision measurements.

\section{ACKNOWLEDGMENTS}

We are grateful for the support of Air Force Office of Scientific Research (Award No. FA-9550-20-1-0366) and the Robert A. Welch Foundation (A-1943-20210327).

\section{APPENDIX}

We give another approach to obtain the QFI in Sec. IV. The steady-state solution is proved to be a pure coherent state $\left|\alpha_{0}, \beta_{0}\right\rangle$. Thus we can apply the simpler method for pure states to find the SLDs and the QFI as introduced by Fujiwara and Nagaoka [45]. For a pure state, the QFI is given by

$$
F_{Q}(\varepsilon)=4\left(\left\langle\partial_{\varepsilon} \psi \mid \partial_{\varepsilon} \psi\right\rangle-\left|\left\langle\psi \mid \partial_{\varepsilon} \psi\right\rangle\right|^{2}\right),
$$


where the state

$$
|\psi\rangle=\left|\alpha_{0}, \beta_{0}\right\rangle=\sum_{m, n} \frac{\alpha_{0}^{m} \beta_{0}^{n}}{\sqrt{m ! n !}} e^{-\frac{\left|\alpha_{0}\right|^{2}+\left|\beta_{0}\right|^{2}}{2}}|m, n\rangle,
$$

in the Fock representation, where we apply the derivatives and obtain

$$
\left|\partial_{\varepsilon} \psi\right\rangle=\sum_{m, n}\left[\left(\frac{m-\left|\alpha_{0}\right|^{2}}{\left|\alpha_{0}\right|} \frac{\partial\left|\alpha_{0}\right|}{\partial \varepsilon}+\frac{n-\left|\beta_{0}\right|^{2}}{\left|\beta_{0}\right|} \frac{\partial\left|\beta_{0}\right|}{\partial \varepsilon}+i m \frac{\partial \theta_{\alpha}}{\partial \varepsilon}+i n \frac{\partial \theta_{\beta}}{\partial \varepsilon}\right) \times \frac{\alpha_{0}^{m} \beta_{0}^{n}}{\sqrt{m ! n !}} e^{-\frac{\left|\alpha_{0}\right|^{2}+\left|\beta_{0}\right|^{2}}{2}}|m, n\rangle\right] .
$$

The terms of the QFI can then be calculated,

$$
\left\langle\partial_{\varepsilon} \psi \mid \partial_{\varepsilon} \psi\right\rangle=\left|\alpha_{0}\right|^{2}\left(1+\left|\alpha_{0}\right|^{2}\right)\left(\frac{\partial \theta_{\alpha}}{\partial \varepsilon}\right)^{2}+\left|\beta_{0}\right|^{2}\left(1+\left|\beta_{0}\right|^{2}\right)\left(\frac{\partial \theta_{\beta}}{\partial \varepsilon}\right)^{2}+2\left|\alpha_{0}\right|^{2}\left|\beta_{0}\right|^{2} \frac{\partial \theta_{\alpha}}{\partial \varepsilon} \frac{\partial \theta_{\beta}}{\partial \varepsilon}+\left(\frac{\partial\left|\alpha_{0}\right|}{\partial \varepsilon}\right)^{2}+\left(\frac{\partial\left|\beta_{0}\right|}{\partial \varepsilon}\right)^{2},
$$

and

$$
\left\langle\psi \mid \partial_{\varepsilon} \psi\right\rangle=i\left(\left|\alpha_{0}\right|^{2} \frac{\partial \theta_{\alpha}}{\partial \varepsilon}+\left|\beta_{0}\right|^{2} \frac{\partial \theta_{\beta}}{\partial \varepsilon}\right) .
$$

We substitute the results to Eq. (A1) and obtain

$$
F_{Q}(\varepsilon)=4\left|\frac{\partial \alpha_{0}}{\partial \varepsilon}\right|^{2}+4\left|\frac{\partial \beta_{0}}{\partial \varepsilon}\right|^{2}
$$

which is the same result as Eq. (24) in the main text.

[1] C. M. Bender and S. Boettcher, Real Spectra in Non-Hermitian Hamiltonians Having $\mathcal{P} \mathcal{T}$ Symmetry, Phys. Rev. Lett. 80, 5243 (1998).

[2] C. M. Bender, Making sense of non-Hermitian Hamiltonians, Rep. Prog. Phys. 70, 947 (2007).

[3] J. Wiersig, Enhancing the Sensitivity of Frequency and Energy Splitting Detection by Using Exceptional Points: Application to Microcavity Sensors for Single-Particle Detection, Phys. Rev. Lett. 112, 203901 (2014).

[4] W. Chen, Ş. K. Özdemir, G. Zhao, J. Wiersig, and L. Yang, Exceptional points enhance sensing in an optical microcavity, Nature (London) 548, 192 (2017).

[5] H. Hodaei, A. U. Hassan, S. Wittek, H. Garcia-Gracia, R. El-Ganainy, D. N. Christodoulides, and M. Khajavikhan, Enhanced sensitivity at higher-order exceptional points, Nature (London) 548, 187 (2017).

[6] R. El-Ganainy, K. G. Makris, M. Khajavikhan, Z. H. Musslimani, S. Rotter, and D. N. Christodoulides, NonHermitian physics and PT symmetry, Nat. Phys. 14, 11 (2018).

[7] Ş. K. Özdemir, S. Rotter, F. Nori, and L. Yang, Parity-time symmetry and exceptional points in photonics, Nat. Mater. 18, 783 (2019).

[8] M.-A. Miri and A. Alu, Exceptional points in optics and photonics, Science 363 (2019).

[9] H. Zhang, R. Huang, S.-D. Zhang, Y. Li, C.-W. Qiu, F. Nori, and H. Jing, Breaking anti-PT symmetry by spinning a resonator, Nano Lett. 20, 7594 (2020).

[10] J. M. P. Nair, D. Mukhopadhyay, and G. S. Agarwal, Enhanced Sensing of Weak Anharmonicities through Coherences in Dissipatively Coupled Anti-PT symmetric Systems, Phys. Rev. Lett 126, 180401 (2021).
[11] P. Peng, W. Cao, C. Shen, W. Qu, J. Wen, L. Jiang, and Y. Xiao, Anti-parity-time symmetry with flying atoms, Nat. Phys. 12, 1139 (2016).

[12] J. H. Wu, M. Artoni, and G. C. La Rocca, Non-Hermitian Degeneracies and Unidirectional Reflectionless Atomic Lattices, Phys. Rev. Lett. 113, 123004 (20014).

[13] J. H. Wu, M. Artoni, and G. C. La Rocca, Parity-timeantisymmetric atomic lattices without gain, Phys. Rev. A 91, 033811 (2015).

[14] X. Wang and J.-H. Wu, Optical $\mathcal{P} \mathcal{T}$-symmetry and $\mathcal{P T}$ antisymmetry in coherently driven atomic lattices, Opt. Express 24, 4289 (2016).

[15] Y.-L. Chuang, Ziauddin, and R. K. Lee, Realization of simultaneously parity-time-symmetric and parity-time-antisymmetric susceptibilities along the longitudinal direction in atomic systems with all optical controls, Opt. Express 26, 21969 (2018).

[16] Y. Jiang, Y. Mei, Y. Zhuo, Y. Zhai, J. Li, J. Wen, and S. Du, Anti-Parity-Time Symmetric Optical Four-Wave Mixing in Cold Atoms, Phys. Rev. Lett. 123, 193604 (2019).

[17] L. Ge, and H. E. Türeci, Antisymmetric PT-photonic structures with balanced positive- and negative-index materials, Phys. Rev. A 88, 053810 (2013).

[18] D. A. Antonosyan, A. S. Solntsev, and A. A. Sukhorukov, Parity-time anti-symmetric parametric amplifier, Opt. Lett. 40, 4575 (2015).

[19] V. V. Konotop, and D. A. Zezyulin, Odd-Time Reversal $\mathcal{P} \mathcal{T}$ Symmetry Induced by an Anti- $\mathcal{P} \mathcal{T}$-Symmetric Medium, Phys. Rev. Lett. 120, 123902 (2018).

[20] F. Yang, Y.-C. Liu, and L. You, Anti- $\mathcal{P T}$ symmetry in dissipatively coupled optical systems, Phys. Rev. A 96, 053845 (2017).

[21] Y. Choi, C. Hahn, and J. W. Yoon, Observation of an anti-PTsymmetric exceptional point and energy-difference conserving 
dynamics in electrical circuit resonators, Nat. Commun 9, 2182 (2018).

[22] Y. Li, Y.-G. Peng, L. Han, M.-A. Miri, W. Li, M. Xiao, X.-F. Zhu, J. Zhao A. Alu, S. Fan, and C.-W. Qiu, Anti-parity-time symmetry in diffusive systems, Science 364, 1261 (2021).

[23] Y. Yang, Y.-P. Wang, J. W. Rao, Y. S. Gui, B. M. Yao, W. Lu, and C.-M. Hu, Unconventional Singularity in Anti-Parity-Time Symmetric Cavity Magnonics, Phys. Rev. Lett. 125, 147202 (2020).

[24] J. W. Wen, G. Qin, and C. Zheng, S. Wei, X. Kong, T. Xin, and G. Long, Observation of information flow in the anti- $\mathcal{P} \mathcal{T}$ symmetric system with nuclear spins, npj Quantum Inf. 6, 28 (2020).

[25] J. Wiersig, Prospects and fundamental limits in exceptional point-based sensing, Nat. Commun 11, 2454 (2020).

[26] R. A. Fisher, On the dominance ratio, Proc. R. Soc. Edinburgh 42, 321 (1922).

[27] H. Cramér, Mathematical Methods of Statistics (Princeton University Press, Princeton, NJ, 1946), Chap. 33.

[28] C. R. Rao, Information and the accuracy attainable in the estimation of statistical parameters, Bulletin. Calcutta Math. Soc. 37, 81 (1945).

[29] C. W. Helstrom, Quantum Detection and Estimation Theory (Academic, New York, 1976), Chap. VIII.4.

[30] A. S. Holevo, Probabilistic and Statistical Aspects of Quantum Theory (North-Holland, Amsterdam, 1982).

[31] S. L. Braunstein and C. M. Caves, Statistical distance and the geometry of quantum states, Phys. Rev. Lett. 72, 3439 (1994).

[32] B. M. Escher, R. L. de Matos Filho, and L. Davidovich, General framework for estimating the ultimate precision limit in noisy quantum-enhanced metrology, Nat. Phys. 7, 406 (2011).

[33] C. L. Latune, B. M. Escher, R. L. de Matos Filho, and L. Davidovich, Quantum limit for the measurement of a classical force coupled to a noisy quantum-mechanical oscillator, Phys. Rev. A 88, 042112 (2013).
[34] B. M. Escher, L. Davidovich, N. Zagury, and R. L. de Matos Filho, Quantum Metrological Limits via a Variational Approach, Phys. Rev. Lett. 109, 190404 (2012).

[35] L. J. Fiderer, J. M. E. Fraisse, and D. Braun, Maximal Quantum Fisher Information for Mixed States, Phys. Rev. Lett. 123, 250502 (2019).

[36] A. N. Jordan, J. M.-Rincon, and J. C. Howell, Technical Advantages for Weak-Value Amplification: When Less is More, Phys. Rev. X 4, 011031 (2014).

[37] Y. Chen and H. Yuan, Maximal quantum Fisher information matrix, New J. Phys. 19, 063023 (2017).

[38] J. Liu, H. Yuan, X. M. Lu, and X. Wang, Quantum Fisher information matrix and multiparameter estimation, J. Phys. A: Math. Theor. 53, 023001 (2019).

[39] A. Monras and M. G. Paris, Optimal Quantum Estimation of Loss in Bosonic Channels, Phys. Rev. Lett. 98, 160401 (2007).

[40] P. Binder and D. Braun, Quantum parameter estimation of the frequency and damping of a harmonic oscillator, Phys. Rev. A 102, 012223 (2020).

[41] G. Adesso, F. Dell'Anno, S. De Siena, F. Illuminati, and L. A. M. Souza, Optimal estimation of losses at the ultimate quantum limit with non-Gaussian states, Phys. Rev. A 79, 040305(R) (2009).

[42] J. Wang, L. Davidovich, and G. S. Agarwal, Quantum sensing of open systems: Estimation of damping constants and temperature, Phys. Rev. Research 2, 033389 (2020).

[43] For the properties of the displacement operators, see, for example, G. S. Agarwal, Quantum Optics (Cambridge University Press, Cambridge, U.K., 2013), Chap. 1.

[44] J. Wang and G. S. Agarwal, Quantum Fisher Information Bounds on Precision Limits of Circular Dichroism, Phys. Rev. A 104, 062613 (2021).

[45] A. Fujiwara and H. Nagaoka, Quantum Fisher metric and estimation for pure state models, Phys. Lett. A 201, 119 (1995). 